\title{
Mixed-Methods Evaluation of the Incorporation of Home Specimen Self-Collection Kits for Laboratory Testing in a Telehealth Program for HIV Pre-exposure Prophylaxis
}

\author{
Emily E. Chasco ${ }^{1}$ (D) Angela B. Hoth ${ }^{1} \cdot$ Hyunkeun $\mathrm{Cho}^{2} \cdot$ Cody Shafer $^{3} \cdot$ Aaron J. Siegler $^{4} \cdot$ Michael E. Ohl $^{1,5}$
}

Accepted: 20 February 2021 / Published online: 19 March 2021

(c) The Author(s), under exclusive licence to Springer Science+Business Media, LLC, part of Springer Nature 2021

\begin{abstract}
Home specimen self-collection kits with central laboratory testing may improve persistence with PrEP and enhance telehealth programs. We offered Iowa TelePrEP clients the choice of using a home kit or visiting a laboratory site for routine monitoring. Mixed-methods evaluation determined the proportion of clients who chose a kit, factors influencing choice, associations between kit use and completion of indicated laboratory monitoring, and user experience. About 46\% (35/77) chose to use a kit. Compared to laboratory site use, kit use was associated with higher completion of extra-genital swabs (OR 6.33, 95\% CI $1.20-33.51$, for anorectal swabs), but lower completion of blood tests (OR 0.21, 95\% CI 0.06-0.73 for creatinine). Factors influencing choice included self-efficacy to use kits, time/convenience, and privacy/confidentiality. Clients reported kit use was straight-forward but described challenges with finger prick blood collection. Telehealth PrEP programs should offer clients home kits and support clients with blood collection and kit completion.
\end{abstract}

Keywords PrEP $\cdot$ HIV prevention $\cdot$ Telehealth $\cdot$ Home-testing

\section{Resumen}

Los kits de pruebas caseras de auto-recolección junto con los ensayos de laboratorio central puedan mejorar la persistencia de PrEP y aumentar los programas de telesalud. Ofrecimos a los clientes de Iowa TelePrEP la opción de o utilizar un kit de pruebas caseras o visitar un sitio de laboratorio para seguimiento rutinario.La evaluación de métodos mixtos determinó la proporción de los clientes que eligieron un kit, los factores que influyen en la elección, las asociaciones entre el uso del kit y la realización del monitoreo de laboratorio indicado y la experiencia de los usuarios. Casi $46 \%$ (35 de 77) eligió utilizar un kit. Comparado con el uso del sitio de laboratorio, el kit fue asociado con mayores tasas de terminación para los hisopos extragenitales (OR 6.33, 95\% CI 1.20-33.51, para hisopos anorectales), pero menores tasas de terminación para los análisis de sangre (OR 0.21, 95\% CI 0.06-0.73 para creatinina). Los factores que influyeron en la elección incluyeron la autoeficacia para usar los kits, el tiempo / la comodidad y la privacidad/ confidencialidad. Los clientes informaron que el uso del kit fue

Emily E. Chasco

emily-chasco@uiowa.edu

1 Department of Internal Medicine, University of Iowa Carver College of Medicine, Iowa City, IA, USA

2 Department of Biostatistics, University of Iowa College of Public Health, Iowa City, IA, USA

3 HIV Special Projects Division, NuCara Pharmacy, Pleasant Hill, IA, USA

4 Department of Behavioral, Social, and Health Education Sciences, Rollins School of Public Health, Emory University, Atlanta, GA, USA

5 Center for Comprehensive Access and Delivery Research and Evaluation (CADRE), Iowa City VA Medical Center, Iowa City, IA, USA 
sencillo pero describieron desafíos con la recolección de sangre por un pinchazo. Los programas de PrEP de telesalud deben ofrecer a los clientes kits para el hogar y apoyarlos con la recolección de sangre y la terminación del kit.

$\begin{array}{ll}\text { Abbreviations } \\ \text { CDC } & \text { Centers for Disease Control and Prevention } \\ \text { CFAR } & \text { Center for AIDS Research } \\ \text { CLIA } & \text { Clinical Laboratory Improvement } \\ & \text { Amendments } \\ \text { CT } & \text { Chlamydia trachomatis } \\ \text { FTC/TAF } & \text { Emtricitabine/tenofovir alafenamide } \\ \text { FTC/TDF } & \text { Emtricitabine/tenofovir disoproxil fumarate } \\ \text { HIV } & \text { Human immunodeficiency virus } \\ \text { IDI } & \text { In-depth interviews } \\ \text { IDPH } & \text { Iowa Department of Public Health } \\ \text { GEE } & \text { Generalized estimating equations } \\ \text { MSM } & \text { Men who have sex with men } \\ \text { NG } & \text { Neisseria gonorrhea } \\ \text { PrEP } & \text { Pre-exposure prophylaxis } \\ \text { PWID } & \text { Person who injects drugs } \\ \text { RPR } & \text { Rapid plasma reagin } \\ \text { STD } & \text { Sexually transmitted disease } \\ \text { UI } & \text { University of Iowa }\end{array}$

\section{Introduction}

Pre-exposure prophylaxis (PrEP) using emtricitabine/tenofovir disoproxil fumarate (FTC/TDF) or emtricitabine/tenofovir alafenamide (FTC/TAF) is a safe and effective method for the prevention of HIV [1-3]. Persistence with PrEP requires quarterly healthcare visits to renew medication prescriptions and obtain routine laboratory monitoring tests [4]. The need for quarterly visits may make it difficult for some clients to persist with PrEP because of long distances to healthcare providers, lack of transportation, challenges of fitting visits around work schedules, and stigma experienced in healthcare settings [5-10].

Direct-to-client telehealth (i.e., serving clients at home or in other non-healthcare settings using videoconferencing and related technology) is a well-accepted method for PrEP care that can overcome barriers related to distance, inconvenience, and stigma [7, 11-13]. Little is known about best practices for incorporating routine laboratory monitoring in direct-to-client telehealth programs, which often serve clients who do not routinely visit healthcare settings and live far from providers. Approaches include arranging for monitoring tests in existing laboratory sites near clients' homes and using home kits that allow clients to self-collect test specimens and mail them to a central laboratory for analysis $[6,12]$.

In 2017, the Iowa Department of Public Health (IDPH) and the University of Iowa (UI) partnered to create the Iowa TelePrEP program to improve access to PrEP in rural and small urban areas [12]. Iowa TelePrEP combined: (1) referral of clients from IDPH's statewide network of HIV and sexually transmitted infection testing sites and Disease Intervention Specialist programs, (2) PrEP counseling and prescribing during direct-to-client telehealth visits conducted by UI pharmacists working within a collaborative practice model, and (3) medication delivery by mail. To test feasibility, the program was initially piloted with 43 clients in a single county health department from February-August 2017. Iowa TelePrEP gradually expanded after the pilot, partnering with additional public health departments in eastern Iowa in 2017-2018 before scaling up statewide in 2019 [12].

Initially, Iowa TelePrEP arranged laboratory monitoring for clients within a network of existing public health and commercial laboratory sites. The overall quality of laboratory monitoring was high using this approach, with $96 \%$ of all indicated blood tests completed [12]. However, only $53 \%$ of indicated pharyngeal and anorectal screening tests for Neisseria gonorrhea (NG) and Chlamydia trachomatis (CT) infection were completed. This was largely because many commercial and local healthcare system laboratory sites were unable to accept client self-collected anorectal and pharyngeal swabs because of protocols prohibiting selfobtained swabs, and lack of staff on site to collect swabs. In addition, some clients reported traveling to public health or commercial laboratory sites was difficult.

In 2018 and 2019, Iowa TelePrEP partnered with the Emory University Center for AIDS Research (CFAR) to offer clients the option of using home specimen self-collection kits ("home kits") with central laboratory testing, instead of traveling to laboratory sites. These kits allowed clients to self-collect blood specimens as well as urine, anorectal swab, and pharyngeal swab specimens for NG/ CT testing [6, 14]. We completed a mixed-methods evaluation of the incorporation of home kits in the Iowa TelePrEP program to guide further improvement and inform similar efforts in other telehealth programs. We aimed to determine: (1) the proportion of clients who chose to use home kits instead of traveling to laboratory sites, (2) factors influencing client choice, (3) laboratory test completion rates using home kits compared to laboratory sites, and (4) client experience using kits.

\section{Methods}

Iowa TelePrEP offered home specimen self-collection kits to clients as a quality improvement initiative. Mixed-methods evaluation of the initiative combined quantitative analysis of data extracted from clinical records routinely generated in 
the TelePrEP program, and qualitative analysis of in-depth interviews with clients offered the option of using a home kit versus traveling to a laboratory site. The Institutional Review Board at UI approved all evaluation activities.

\section{Description of Home kit Initiative}

Iowa TelePrEP used internal program funds to procure 80 home kits, a service provided by the Emory CFAR. Clients received kits from a CFAR-contracted Amazon Distribution Center and were not charged for kits or test completion. Kits included: (1) microcapillary tubes for blood collection for HIV tests, creatinine, and semi-quantitative rapid plasma reagin (RPR) tests for syphilis, (2) urine collection for NG and CT testing, and (3) swabs for self-collection of pharyngeal and anorectal specimens for NG and CT. Printed instructions described self-collection of urine specimens and pharyngeal and anorectal swabs, as well as collection of blood in microcapillary tubes (later replaced by gravity tubes) after a finger prick with subsequent transfer of blood to a larger tube for transport (see appendices for kit contents and instructions). Clients returned kits by FedEx to a Clinical Laboratory Improvement Amendments (CLIA)-certified laboratory for analysis, with reporting of results directly to a TelePrEP pharmacist using a secure online portal. The RPR assay used to test for syphilis was validated in 2005 per standard CLIA-waiver required procedures; further details of the laboratory tests performed have been described elsewhere [6].

From July 2018 to May 2019, pharmacists offered a consecutive series of clients due for quarterly follow up visits the choice to use a home kit or to travel to a public health or commercial laboratory site for testing, as they had previously done. During videoconference or telephone/email contacts with individual clients, pharmacists described the kit process, provided information on estimated travel time to the nearest laboratory site, and answered questions. They then arranged to mail kits to clients or ordered tests at a laboratory site, per client preference. Pharmacists did not offer kits to new clients completing baseline labs or to cisgender female clients because of their need for additional monitoring tests not included in the kit (e.g. quarterly pregnancy tests). Pharmacists continued to offer home kits at each quarterly visit until kits ran out. Clients used an average of 2 kits (range 1-4) and resumed travel to laboratory sites when kit supply ran out.

\section{Description of Home Kit Evaluation}

\section{Quantitative Analyses}

We collected data on clients beginning three months prior to first home kit use and continuing six months following final kit use; quantitative data collection therefore spanned the period from April 2018 to October 2019. We reviewed pharmacist notes to determine whether individual clients chose to use home kits or laboratory sites, and client characteristics including age, self-reported gender (male, Trans*man, Trans*woman, gender fluid/queer, non-binary, or other), self-reported race/ethnicity (Black, White, Latino, Asian), PrEP indication, rural versus urban residence, and estimated client travel time (in minutes) one-way to the nearest laboratory site. We classified PrEP indications following CDC PrEP guidelines [4] as: (1) man who has sex with men (MSM) with risk factor(s) (e.g., anal sex without a condom with a partner with known or possible HIV infection, or bacterial sexually transmitted infection in the past six months); (2) heterosexual man with risk factor(s) (e.g., vaginal or anal sex without a condom with a partner at substantial risk for HIV infection, sex with a partner who is HIV-positive, or history of syphilis or gonorrhea in the past six months); or (3) person who uses intravenous drugs with needle sharing. We defined client residence as urban (metro counties with population $\geq 50,000$ ) or rural (non-metropolitan counties population $<50,000$ ). We determined the number and percentage of clients who chose to use a home kit versus a laboratory site and compared characteristics of clients choosing home kits versus laboratory sites using Fisher's Exact or chi-square tests for categorical variables and Mann-Whitney $\mathrm{U}$ tests for continuous variables.

We conducted within-person comparisons of test completion rates using home kits versus laboratory sites among clients who chose to use a home kit, and who had used both a kit and a laboratory site at least once for routine PrEP monitoring. For each client, this included episodes completed at a laboratory site prior to offer to use a home kit, each episode while using a home kit, and at least one episode using a laboratory site again after home kits were no longer available. For each laboratory test during each monitoring episode, we created a binary variable indicating test completion leading to a usable result, coded 1 if specimen was submitted and test result usable, otherwise 0 . Separate variables indicated completion of: (1) HIV testing from blood, (2) creatinine from blood, (3) RPR from blood, (4) NG/CT from urine, (5) NG/CT from pharyngeal swab, and (6) NG/CT from anorectal swab. Pharmacists ordered variable numbers of tests at each laboratory site episode according to client needs (i.e., quarterly HIV and RPR tests, creatinine every three or six months depending on client risk factors for kidney disease, and quarterly urine, pharyngeal, and anorectal NG and CT tests based on sexual histories), but ordered all tests at each monitoring episode using home kits because it was not possible to select individual tests from kits for performance. Only ordered tests were included in analyses. We verified there were no meaningful differences in test completion rates at laboratory sites before and after home kit use, suggesting 
there were no secular trends or maturation effects in test completion rates over time that could bias results.

We classified tests as incomplete (i.e., coded 0 ) if the necessary specimen was not obtained or a usable result could not be reported because of specimen deficiencies, such as an inadequate volume of blood or swab transport media. For home kits, lab tests were coded as completed only if it was completed by returning the kit with a usable result. If a client accepted the offer of a kit and it was sent, but the client then went instead to a laboratory site for their tests, this was coded as incomplete (i.e., coded 0). Pharmacists documented reasons for incomplete tests using home kits (e.g., kit not returned or inadequate sample) and laboratory sites (e.g., specimen mishandled at lab or not obtained despite order) and contacted affected clients to arrange for repeat testing in a laboratory site to ensure appropriate PrEP care, but these repeated tests were not included in analyses. For both clients using home kits and clients using laboratory sites, Iowa TelePrEP protocol required an HIV test every three months and a creatinine at least every six months to renew PrEP prescriptions, but every effort was made to work with clients to obtain these tests and avoid lapses in PrEP.

We calculated the proportion of tests completed for each test type, by use of home kit versus laboratory site, using the laboratory monitoring episode as the unit of analysis with multiple episodes clustered within clients. We used logistic regression to determine within-client associations between use of home kit versus laboratory site (the independent variable) and test completion (the dependent variable), adjusting for client characteristics (age, race/ethnicity, rural versus urban residence, and travel time to laboratory site). We used Generalized Estimating Equations (GEE) to account for clustering of multiple monitoring episodes within individuals over time, under a first-order autoregressive working correlation structure. We estimated odds ratios for each comparison, with Bonferroni corrected 95\% confidence intervals given multiple comparisons for the six test types. Analyses were completed using SAS software v9.4 [15] and R software 3.6.2 [16].

\section{Qualitative Analyses}

We conducted semi-structured in-depth interviews (IDI) by phone with TelePrEP clients offered the option of using a home kit versus traveling to a laboratory site between May 2019 and March 2020. All participants provided informed consent prior to participating in interviews. We recruited IDI participants purposively to achieve balanced representation from both clients who chose to use a kit and clients who chose to visit a laboratory site. The interview guide was informed by literature on the PrEP care cascade and barriers to PrEP use, as well as informal client feedback shared with the TelePrEP pharmacist in the course of routine PrEP care during Iowa TelePrEP's pilot and initial expansion phases. Interview questions elicited client perspectives on the home kit offer, factors influencing their choice to use or not use a kit, experiences at laboratory sites and in PrEP care, experiences using home kits, and feedback for quality improvement. Interviews were audio-recorded with one exception due to recorder failure; the interviewer took written notes documenting participant responses instead. Recordings were transcribed, and transcripts and notes uploaded to MAXQDA [17] qualitative data management software for analysis.

We conducted a template analysis $[18,19]$ of home kit evaluation data led by a qualitative researcher on the study team. Each transcript was read in its entirety and a memo created summarizing key findings and contextual information related to the aims of the home kit evaluation. A preliminary a priori code list was developed from the interview guide structure, summary memos, and previous client and staff feedback. The qualitative researcher applied this code list to a subset of 6 transcripts (30\%; 3 kit users, 3 nonusers), with new themes added as they emerged from the data. Next, the revised code list was used to create a hierarchical template with 6 top-level themes: (1) Initial perceptions of home kit offer, (2) Factors influencing choice, (3) Laboratory site use experiences, (4) Home kit use experiences, (5) Perceived benefits of home kits versus laboratory sites (post-kit use), and (6) Future kit use and quality improvement. The provisional template was tested on the remaining 15 transcripts and refined in an iterative process. The final template was then applied to the full set of transcripts. Throughout the analysis, the qualitative researcher met with the study team and Iowa TelePrEP staff to share preliminary findings. These meetings provided opportunities for discussion, for staff to share their professional expertise and insight, and encouraged consensus on data interpretation. Final template structure including top- and second-level themes can be found in Fig. 1; we focus primarily on findings from (2) Factors influencing choice and (4) Home kit use experiences here, with qualitative evidence from other top-level themes included where relevant.

\section{Results}

\section{Proportion and Characteristics of Clients Choosing to use Home Kits Versus Laboratory Sites}

Pharmacists offered home kits to 77 clients, 35 (45.5\%) of whom chose to use a kit instead of a laboratory site (Table 1). All clients offered kits self-identified their gender as male, and none as Trans*man, Trans*woman, gender fluid/queer, non-binary, or other. Most clients offered kits lived in urban areas (81.8\%) and within 15 min of a 
Fig. 1 Final template, including top- and second-level themes
1. Initial perceptions of home kit offer

1.1 Questions or concerns

1.2 Decision to accept or refuse

2. Factors influencing choice

2.1 Self-efficacy

2.2 Time/convenience

2.3 Privacy/confidentiality

2.4 Method of delivery

2.5 Cost considerations

3. Laboratory site user experience

3.1 Identifying a laboratory site for PrEP monitoring

3.2 Location and distance to laboratory site

3.3 Service availability

3.4 Time commitment

3.5 Lab order processes

3.6 Staff interactions

3.7 Insurance coverage and lab fees

3.8 PrEP monitoring and test results

4. Home kit user experience

4.1 Receipt of kit

4.2 Review of kit instructions

4.3 Specimen collection

4.4 Return of kit

5. Perceived benefits of home kits versus laboratory sites (post-kit use)

5.1 Risks related to privacy, confidentiality, and stigma

5.2 Relative cost burden

5.3 Comprehensiveness of available tests

5.4 Convenience

6. Future kit use and quality improvement.

6.1 Learning curve and repeat kit use

6.2 Preferences for future PrEP monitoring

6.3 Feedback for home kit improvement laboratory site $(81.0 \%)$. Characteristics of clients who chose to use home kits versus a laboratory site were overall similar with regard to age, race/ethnicity, rurality, travel time to nearest laboratory site, and PrEP indication (Table 1). No differences were statistically significant in this small sample.

\section{Completion of Laboratory Monitoring using Home Kits Versus Laboratory Sites}

Clients who chose to use a home kit had 207 laboratory monitoring episodes during the evaluation period, including
79 episodes using a home kit and 128 using a laboratory site (Table 2). HIV tests were done and usable for 66 (83.5\%) kit episodes versus $128(100.0 \%)$ lab episodes, creatinine for 64 $(81.0 \%)$ kit and 91 (91.9\%) laboratory episodes, and blood RPR for 66 (83.5\%) kit and 121 (94.5\%) laboratory episodes. Reasons for incomplete blood tests with kits were: kit not returned (7), returned with no blood specimen (1), and insufficient blood sample (5) to complete some or all blood tests.

Using kits, NG and CT tests were completed for 71 (89.9\%) of 79 episodes for urine, pharyngeal, and anorectal specimens, regardless of specimen type. This compared 
Table 1 Client characteristics by choice to use home kit

\begin{tabular}{|c|c|c|c|c|}
\hline Characteristic & Accepted kit $(\mathrm{N}=35)$ & Refused kit $(\mathrm{N}=42)$ & Test statistic & $\mathrm{p}$ \\
\hline Age, years, mean (range) & $32(20-64)$ & $33(19-60)$ & $734^{\mathrm{c}}$ & $0.99, \mathrm{NS}$ \\
\hline Race/ethnicity, N (\%) & & & $0.26^{\mathrm{b}, \mathrm{d}}$ & $0.61, \mathrm{NS}$ \\
\hline White & $30(85.7)$ & $33(78.6)$ & & \\
\hline Black & $4(11.4)$ & $3(7.1)$ & & \\
\hline Latino & $1(2.9)$ & $2(4.8)$ & & \\
\hline Asian & $0(0)$ & $4(9.5)$ & & \\
\hline PrEP Indication, N (\%) & & & $0.50^{\mathrm{e}}$ & $0.50, \mathrm{NS}$ \\
\hline $\mathrm{MSM}+$ risk factors & $35(100)$ & $40(95.2)$ & & \\
\hline Heterosexual man + risk factors & $0(0)$ & $2(4.8)$ & & \\
\hline PWID $^{\mathrm{a}}$ & 0 & $0(0)$ & & \\
\hline Residence, N (\%) & & & $0.01^{\mathrm{d}}$ & $0.94, \mathrm{NS}$ \\
\hline Urban & $29(82.9)$ & $34(81.0)$ & & \\
\hline Rural & $6(17.1)$ & $8(19.0)$ & & \\
\hline Travel time to lab, minutes, mean (range) & $12(2-44)$ & $19(1-105)$ & $565^{\mathrm{c}}$ & $0.08, \mathrm{NS}$ \\
\hline
\end{tabular}

${ }^{\mathrm{a}} \mathrm{PWID}=$ person who injects drugs

${ }^{\mathrm{b}}$ White versus Nonwhite

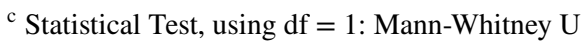

${ }^{\mathrm{d}}$ Statistical Test, using df $=1$ : Chi-square with Yates correction

${ }^{\text {e}}$ Statistical Test, using $\mathrm{df}=1$ : Fisher's exact test

to 117 (91.4\%) of 128 indicated urine samples, 68 (61.3\%) of 111 anorectal swabs, and $72(56.3 \%)$ of 128 pharyngeal swabs using laboratory sites. NG and CT tests were incomplete for seven kits not returned and one kit returned with blood sample only. Low rates of pharyngeal and anorectal testing in laboratory sites were related to many sites not allowing submission of client self-collected swabs.

In adjusted within-client comparisons using logistic regression and GEE (Table 2), use of kits was associated with higher odds of completing NG and CT tests for anorectal (OR 6.33, 95\% CI 1.20-33.51) and pharyngeal swabs (OR 5.96, 95\% CI 1.16-30.68) compared to laboratory site testing. In contrast, use of kits was associated with lower odds of completing blood tests, including creatinine (OR $0.21,95 \%$ CI $0.06-0.73$ ) and syphilis testing (OR $0.23,95 \%$ CI 0.06-0.86). We could not fit regression models for HIV testing because all (128 of 128) HIV tests were complete at laboratory sites, but proportion completing HIV tests and syphilis tests were similar using home kits $(83.5 \%$ versus $81.0 \%)$.
Table 2 Completion of monitoring tests by use of home kit versus laboratory site, among clients using both kits and laboratory sites over time

\begin{tabular}{|c|c|c|c|c|c|c|}
\hline \multirow[t]{2}{*}{ Monitoring test } & \multicolumn{2}{|l|}{ Home kit } & \multicolumn{2}{|c|}{ Laboratory site } & \multirow[t]{2}{*}{$\mathrm{OR}^{\mathrm{a}}(95 \% \mathrm{CI})$} & \multirow[t]{2}{*}{$\mathrm{p}$} \\
\hline & $\mathrm{N}$ eligible & $\mathrm{N}(\%)$ complete & $\mathrm{N}$ eligible & $\mathrm{N}(\%)$ complete & & \\
\hline HIV & 79 & $66(83.5)$ & 128 & $128(100)$ & $* * \mathrm{~b}$ & $* *$ \\
\hline Creatinine & 79 & $64(81.0)$ & 99 & $91(91.9)$ & $0.21(0.06-0.73)$ & 0.01 \\
\hline Syphilis (RPR) & 79 & $66(83.5)$ & 128 & $121(94.5)$ & $0.23(0.06-0.86)$ & 0.01 \\
\hline NG/CT-urine & 79 & $71(90.0)$ & 128 & $117(91.4)$ & $0.63(0.12-3.27)$ & 0.44 \\
\hline NG/CT-anorectal & 79 & $71(90.0)$ & 111 & $68(61.3)$ & $6.33(1.20-33.51)$ & 0.01 \\
\hline NG/CT_-pharyngeal & 79 & $71(90.0)$ & 128 & $72(56.3)$ & $5.96(1.16-30.68)$ & 0.01 \\
\hline
\end{tabular}

The unit of analysis is the laboratory monitoring episode, with multiple episodes clustered within clients who used both laboratory sites and kits over time. This leads to a larger sample size in this analysis than in Table 1. Analyses used logistic regression and generalized estimating equations (GEE) to account for clustering of episodes within clients. See methods

${ }^{a}$ Odds ratio for test completion using home kit compared to laboratory site

${ }^{\mathrm{b}}$ Analysis could not be performed for HIV test because all outcomes in lab were complete. See results 


\section{Factors Influencing Choice to use Kits Versus Laboratory Sites}

Twenty-one clients completed in-depth interviews, ten who chose to use a home kit and eleven who chose a laboratory site. IDI participant-identified factors influencing choice fell primarily under three main themes: self-efficacy to use kits, time/convenience, and privacy/confidentiality concerns. Factors influencing choice grouped by theme and illustrative quotations for each can be found in Table 3 .

Nearly three-quarters of all IDI participants (including 6 kit users and 9 non-users) described factors related to selfefficacy - their perceived ability to complete the home kit process at the time the offer was made - as influential in their decision. Self-efficacy related to perceived ability to complete the kit in a timely manner, follow instructions correctly, and collect required specimens. IDI participants who doubted their ability to complete the kit in a timely manner and were concerned this could delay medication fills refused the offer of a kit. Prior relevant experience, for example professional training in a healthcare field or having a diabetic family member, was sometimes cited as positively influencing perceived ability to complete blood collection.

IDI participants overall expressed high degrees of confidence in their ability to collect pharyngeal and anorectal self-swabs in part due to previous experiences with selfcollection at laboratory sites, and none cited these as their reason for refusing a kit. In contrast, concerns about the blood collection process were common including having to prick one's own finger, the sight of blood, and potentially following directions incorrectly thereby affecting test accuracy. Two IDI participants believed themselves incapable of blood collection and refused a kit for that reason alone, however, IDI participants who accepted a kit were willing to try it despite reservations. For most IDI participants this was one factor among several and it was the balance of factors that tilted their decision to accept or refuse.

Several IDI participants reported initial misconceptions about the amount of blood they would be required to collect, based on blood draw procedures they underwent at laboratory sites. One IDI participant asked the pharmacist about this at the time of offer; the pharmacist clarified the kit procedures and the participant accepted a kit. However, other IDI participants who refused a home kit implied they were giving voice to this confusion for the first time during the interview itself. This indicates that clarifying the blood collection method, a finger prick from an automated device, may be important when offering home specimen self-collection.

Factors under the theme of time/convenience included frequency of laboratory site visits, travel time to sites, client work travel, and schedule convenience. IDI participants who refused a home kit were less likely to view laboratory site visits as burdensome or disruptive to their schedule, had a laboratory site near their home or daily route, and felt the process was easy and quick. IDI participants who accepted the kit were more likely to view travel to a laboratory site as a burden, even if actual travel time was brief. They were also more likely to mention having to schedule an appointment, limited laboratory hours, interruptions to their work or class schedule, or being "busy" as reasons to forego laboratory sites in favor of kits. This supports our finding that calculated travel time was not significantly different in quantitative comparisons of kit users and non-users; impact on client schedule and competing time demands may moderate whether clients perceive travel time to a laboratory site as a barrier. Several IDI participants also cited frequent work travel as influencing their decision to accept or refuse a kit and felt that offering multiple options to complete laboratory monitoring gave clients flexibility to choose what worked best as their situations evolved.

Factors related to the theme of privacy/confidentiality were of significant concern to IDI participants who reported being "out" regarding their sexual orientation, sexual behavior, or PrEP use to differing and context-dependent (e.g., social group versus at work) degrees. Clients discussed coming out as a process rather than a one-time decision, and few IDI participants reported they were fully out about their sexual orientation and sexual behavior in all aspects of their lives. Rather, both where IDI participants were in the process and what they had previously experienced influenced whether they perceived in-person laboratory testing or a home kit as the greater threat to any privacy or confidentiality concerns they had. For example, two IDI participants living with individuals unaware of their sexual behavior and/ or PrEP status viewed this as an impediment to kit use and declined the offer of a home kit. Home kits posed less of a threat to clients who lived alone and therefore had a private location to collect specimens, but one reported he declined a kit because he had already experienced a privacy violation when a visiting guest found his discarded bottle of Truvada in the trash therefore he didn't want the kit lying around his home.

Navigating privacy and confidentiality in PrEP care could be complicated for reasons other than who was present in the home, for example if a client had insurance coverage through a parent, shared a provider with their spouse/partner, or were required to use specific employer-associated laboratories or pharmacies, all situations reported by participants in our study. Some clients also reported significant life changes while in PrEP care, such as a move or enrollment at a college or university, that altered their proximity to a preferred laboratory site or impacted their perceived privacy at home. The diversity of client experiences indicated that whether a client was out did not neatly align with the decision to accept or decline the offer of a home kit. Rather, IDI 
Table 3 Factors influencing client home kit decision from analysis of in-depth interviews $(\mathrm{N}=21)$

\begin{tabular}{lll}
\hline Main theme & Decision factor & Illustrative quotations \\
&
\end{tabular}

Self-efficacy

Perceived ability to complete kit in a timely manner I was concerned about, okay well I get this, I'm gonna remember to do it right now or will it sit there and then, oh crap you know, I'm gonna be late for my fills. Things like that.-Refused Kit

It's also sometimes easier for me, like if I had it at home, I imagine I'd like look at it for a few days. Whereas like if I forced myself to go to the lab I know it would be done.-Refused Kit

Perceived ability to follow instructions correctly

Perceived ability to collect required specimens

Prior relevant experience

Time/convenience

Frequency of laboratory site visits
And I don't know, I guess the test kit, it felt like probably it might have a lot of parts to it, and instructions, and, like I don't even like, when I bleach my hair, I don't even like reading those directions.-Refused Kit

Yeah, I was a little afraid that I'd mess something up or, misread some of the instructions but I ended up getting it figured out.Accepted Kit

Umm, but outside of that the only thing that I was worried about was just getting out my own blood. Not that I'm squeamish or anything, but just I was you know thinking, "How does that work? You prick your finger, you put it in, how do you?" You know, it was just that whole thing. I wanted to make sure that especially that part was accurate enough.-Accepted Kit

Yeah, "cause I cannot stand blood. So I would probably go get roadkill, and test the roadkill for blood before I'd ever stick myself with something to witness blood.-Refused Kit

At first it was a little concerning because I know that um, like when I go in to get my full panel done they pull three whole vials of blood out, and I was like, "How much blood am I gonna have to draw?"-Accepted Kit

So I've kind of done, I went to the [laboratory site] once and they actually, you know, did the test pretty much yourself. The only thing that they did was the, you know, blood retraction.Accepted Kit

No, I mean honestly half of the labs, like half of the times I would go do labs I had to do it myself anyways. It would literally be like a baggy of stuff and say, "All right, go into the restroom."Refused Kit

My dad was diabetic and so I, you know, would, you know I've used lancets before and that kinda thing. And so it doesn't make me squeamish or anything like that, so.-Accepted Kit

It was both a little bit of anxious but also kinda just um, excited because I'm going into the healthcare field so I was really anxious to see the ways that they were gonna test all this stuff too, so.- Accepted Kit

Like basically what I tell myself, my cousin is diabetic and I watched him sticking himself like every day, multiple times a day. And I'm like, "I could do this once every three months." Like, "You need to get over it." But at the same time uh, yeah... that was just my deal, is I figured I'll just go in and have it done.-Refused Kit

And so I was going to [laboratory site] and they were doing the STD tests at no charge. And then I would have to set up a second appointment, um and take off work to go to the lab, to get the blood work done.-Accepted Kit

No, I just get tired of going in, especially with [non-PrEP monitoring] tests. I'm so used to them poking my arms, I just get tired of it.-Accepted Kit 
Table 3 (continued)

\begin{tabular}{lll}
\hline Main theme & Decision factor & Illustrative quotations \\
\end{tabular}

Travel time to laboratory sites

Client work travel

Schedule convenience

Privacy/confidentiality (Not) Being “out” regarding sexual orientation, sexual behavior, and PrEP use

Perceived risks associated with kit use relative to laboratory site use
Also, you know to be honest, it has to do with where I live. I live...close to the hospital. Now if I, it was more of a commute or more of an inconvenience, or I lived outside of the city, then yeah, I probably would think twice about it.-Refused Kit

Like I'm willing to go out of my way for certain, like not really go out of my way, but I'm willing to just do certain things myself or go places and do things myself.--Refused Kit

And I may not have time to drive all the way out to um, even if it's only ten minutes, that's ten minutes out, sit there for a half hour, and then ten minutes back. Versus I can do an at-home test kit between classes and send it back in.-Accepted Kit

I was offered the option but I never actually did a home testing kit because again, the hardest part about PrEP was just like, I was never in the same place for a very long time.- - Refused Kit

I may only be around one week of the month, so I have to plan in advance when I will go to the lab.-Accepted $\mathrm{Kit}^{\mathrm{b}}$

I drive right past the lab almost every day you know, so...they're never busy. You know, I don't know if I've ever had to wait.Refused Kit

Which was a lot easier than calling and scheduling an appointment, um, that extra time. And since the clinic was only open from eight to four-thirty, I could do the at-home kit when I got home from classes or anything else I had going on.-Accepted Kit

So, I mean you know, you can do it in the convenience of your own home, so you don't have to make an appointment.Accepted Kit

And then as far as my privacy here...no one really goes to the area of my house like where, where like my prescription is, so. And that's kind of on purpose. Like I really don't want people to, I don't want guests like finding it or something I suppose, so. That's kind of a, yeah, I do kind of think about that a little bit.-Refused Kit

Not difficult, just discretionary issues that I was afraid of. You know, running through my insurance, uh, the company I worked with finding out about it.- - Refused Kit

Insurance at first... because at the time I was on my dad's insurance and he didn't fully know everything, so it was kinda one of those, "How can I go about this without, you know, stuff coming up on the bill?"-Accepted Kit

Not my family. My friends only.- - Refused Kit

'Cause I just didn't want it around the house, didn't want any chance of anything being left to be discovered, so I just wanted to go to a lab and have everything done.--Refused Kit

I'm sharing a place with a roommate, you know, so it's like I don't, yeah. It'll be easier just to go to, you know, the lab.Refused Kit

I think it just came in a pretty, you know, white box. And at the time, I was living alone. I live alone in my house, so just leave at the front door. Nobody knows what it is. Whether it's that or a package from Amazon or wherever, no one knows what's in the package.-Accepted Kit 
Table 3 (continued)

\begin{tabular}{ll}
\hline Main theme & Decision factor \\
\hline Perceived stigma experienced at laboratory sites
\end{tabular}

Method of delivery

Access to mail

Security and reliability of mail

Cost considerations

Cost of kit

Fees associated with laboratory site testing
Illustrative quotations ${ }^{\mathrm{a}}$

And even as prevention, there's still a stigma going in, I think. I mean I'm over the stigma. Like I don't care, it is what it is. 'Cause I know it's preventive and I know that I'm being proactive to this. But I still think the phlebotomist, uh, there's kind of a stigma when they're asking, you know, for an HIV test and this test and this test and... - Accepted Kit

I remember one time I went to get tested at my main care provider there. It was just a super awkward experience all around.Accepted Kit

The first couple times I went there, the two women that drew my blood they just, it might've been, it might've been three times even, um, they kinda like loudly, you know were like, "Oh so you're getting tested for HIV," and loudly saying my name.Refused Kit

Getting to a lab, because I could go anywhere, that made it easier... having things mailed to me, that was the hard part.Refused Kit

And then also a personal circumstance is that the apartment that I live at, like with the mail, like if it was a larger package, they put it in the main office and, long story short, I was just concerned I wasn't gonna be able to access it. 'Cause they're only open during the normal business hours and I tend to be gone early and then home late.-Refused Kit

So like people steal mail and stuff sometimes too, um, or sometimes packages get lost or, there's a lot that goes wrong.Refused Kit

So, the initial reason was because um, 'cause I'm all about costs... when [TelePrEP pharmacist $]$ said that there was a kit that I could just do at home and it was no charge for all of it, I was like well that kills two birds with one stone, so-Accepted Kit

And I just asked her if there was a cost associated and she said there wasn't. So I said, “Okay, why not? I'll just try it out.”Accepted Kit

My labs are considerably more money to go to a lab and have them draw the blood and do those tests, are considerably more expensive. I mean there is some costs to that for me.-Accepted Kit

Also, any time I went to the [health clinic], a lot of their services are free but times you have to draw blood at the lab there's a small fee that they just charge to your bill and um, not a fee that would like prevent me from needing to do that, just a fee that (sighs), if they didn't charge that fee I could forget about it.Accepted Kit

STD Sexually transmitted disease

${ }^{a}$ Quotations have been lightly edited to remove word repetitions (e.g., stammering) and verbal hesitations (e.g., "um"), and to protect confidentiality

${ }^{\mathrm{b}}$ Specific quotation is from written interview notes and not verbatim

participants with privacy/confidentiality concerns weighed potential threats posed by each option within the context of their own unique circumstances and chose the option that mitigated these concerns.

IDI participants shared perceived stigma experienced with healthcare providers in the past, in contrast to more positive experiences with Iowa TelePrEP. This stigma manifested as providers holding different values, or as reluctance to discuss sexual orientation and behaviors, ignorance about PrEP and LGBTQ health, or promotion of abstinence rather than risk reduction for HIV. Some IDI participants also shared stereotypes they had encountered, or even believed themselves, regarding the "type" of person who uses PrEP. Concerns about HIV, sexual orientation, or PrEP-related stigma were reinforced in some cases at laboratory sites where IDI participants reported instances when staff listed 
off or asked the purpose of laboratory orders in front of others, or assumed clients were HIV positive. IDI participants who had had such encounters, or who were not open about their sexual orientation, typically viewed home kits as a more private, confidential option, with a lower risk of stigma.

Other factors were less important among IDI participants we interviewed but might be explored further in future studies. The method of delivery, by mail, was a concern for some who declined a kit, either because of frequent work travel or access to their building's mail room at limited times. Only three IDI participants reported considering relative cost of a home kit (free in this initiative) to using a laboratory site and its associated fees. All three chose to use a kit. However, nearly all IDI participants described cost concerns when considering starting PrEP, and an increase in cost or loss of insurance coverage was commonly cited as a potential reason to stop PrEP in the future. It may be that more IDI participants considered this factor in their decision to accept or refuse a kit but having already discussed general concerns about the cost of PrEP care did not specifically reference it in relation to their home kit decision.

\section{Home Kit User Experiences}

We asked IDI participants who chose to use a home kit $(\mathrm{N}=10)$ about their experiences with kit use and feedback for quality improvement related to receipt of kit, review of kit instructions, specimen collection, and kit return (themes with illustrative quotations are provided in Table 4). IDI participants reported receiving the kit in the mail was straightforward and convenient and had no suggestions for improvement. Those living in a multi-dwelling unit (e.g., apartment, dorm room) did have to retrieve the kit from a front desk or mail room but none considered this a major inconvenience or a threat to privacy. One IDI participant experienced a shipping delay in kit arrival but the TelePrEP pharmacist was able to remedy this before it impacted the participant's next medication fill.

Feedback on kit instructions and specimen collection was mixed. No issues with urine specimen or pharyngeal or anorectal swab collection were reported, and all IDI participants described the instructions generally as "easy to follow," "straight-forward," and written so the user "took it one test at a time." However, IDI participants expressed frustrations with the blood collection process, perceiving it as the most difficult kit activity to complete (even by two who had previous relevant finger prick experience). IDI participants specifically reported difficulty using the microcapillary tube to collect blood from their fingertip for transfer to a larger tube. Following discussion with pharmacists and the study laboratory, it was determined that it was simpler to transfer blood drops directly from the fingertip into the tube until subsequent kits could be sent out with gravity tubes instead, and the collection process was modified accordingly. Three kit users did have difficulty pricking their own finger, but this was not strong enough to deter kit use and they were ultimately successful in doing so.

IDI participants characterized kit return as convenient and easily incorporated into daily routines, describing dropping kits at shipping locations near work or home, or while running errands. Two did share experiences in which shipping delays potentially impacted their test results (one returned their kit a day later than planned due to a closed shipping location, while the other mailed their kit on time but it took longer than expected to arrive at the lab). In both cases, the IDI participants communicated with the TelePrEP pharmacist about the delay, and in the latter case the pharmacist arranged for the client to repeat monitoring at a laboratory site when their kit test results were unusual. Following additional pharmacist guidance, this client then researched pickup times for his local FedEx drop box and planned future kit return to minimize shipping time and potential delays.

\section{Discussion}

We conducted a mixed-methods evaluation of a quality improvement initiative that incorporated home specimen self-collection kits with central laboratory testing in a statewide telehealth program for PrEP delivery. Roughly half of clients offered a kit accepted (45.5\%), indicating that home kits were not a preferred option for some TelePrEP clients. Use of home kits instead of a laboratory site was associated with a higher likelihood of completion of anorectal and pharyngeal testing for sexually transmitted infections, but a lower likelihood of test completion for blood specimens (i.e., HIV, creatinine, and blood RPR). The majority of noncompleted blood specimens resulted from non-return of the kit, indicating that retention efforts and mechanisms to simplify kit completion will be key drivers of home kit use in the future.

The Iowa TelePrEP home kit initiative and evaluation were novel in several ways. First, the initiative was implemented pragmatically in a real-world PrEP care setting, using existing program clients, care protocols, and standard of care delivery. Second, the home kit was added as an additional component to an existing telehealth pharmacistdelivery model designed to address PrEP access barriers in a largely rural and small urban state. Third, we utilized a mixed-methods evaluation approach, using quantitative measures of uptake and quality, and qualitative methods to identify and contextualize client decision and to understand client kit use experience. 
Table 4 Home kit user experience themes from analysis of in-depth interviews $(\mathrm{N}=10)$

\begin{tabular}{ll}
\hline Stage of process & Theme \\
\hline Receipt of kit & Home delivery process was convenient
\end{tabular}

Review of kit instructions Instructions were generally clear and helpful

Blood collection instructions could be confusing or difficult to follow

Existing communication channels provided opportunities to update instructions as needed

Specimen collection

Urine and swab collection were perceived as easy to do

Blood collection was perceived as the most difficult specimen to collect

Updated blood collection instructions improved the collection process for clients
Illustrative quotations $\mathrm{s}^{\mathrm{a}}$

And it was so nice, you got the email saying you got a FedEx package delivered and you knew what it was

Convenience for one of them, just 'cause it got mailed to my hall office and I picked it up, and it took me fifteen minutes, maybe a half hour, to do all the samples

I would read through them, and I mean after the first time I was like, "Oh okay, got it." Clean everything and you know, go from there

I was a little scared that I was gonna do one of them wrong. Or, I was like, "Ooh, lotta options to possibly fail here." But all of the directions were very straightforward, walked you very well through it

I think the first time that I did the blood, that one was a bit confusing just because there were a whole lot of steps with it

The only one that was kinda irritating was the blood collection because the instructions that were sent in the kit wasn't actually what the TelePrEP team wanted us to do

Yeah, it was something with the blood draw. How there's some capillary tube that didn't work really well and she recommended to just drip it directly into the vial instead of using the tube

She thought it might be related to a delay in them receiving the sample. Or testing it. So I volunteered to go to the in-person lab and do it again, and the results came back fine. But then the next time I did the home test kit, there were more clear instructions about how soon it had to get in the mail

And all of the tests were like, once you kind of took it one test at a time, it was all super easy. Like you really couldn't mess it up that hard

Yeah, I did them all every time, and I found it easier to do the throat swab on myself than to have someone else do it

Oh no, those were pretty simple

The blood draw was a little difficult 'cause I've never had to use a lancet on myself. And it was just getting myself to do that and purposely harm myself...Put me a little on edge a little at first but I got through it

And they were slightly more cumbersome to use than going, having a professional phlebotomist take the blood

I wasn't afraid to prick my finger and draw my own blood, but it was, I didn't wanna like go through of all this effort to send it in and be like, "Hey you didn't do it right, you gotta redo it

No, I think the first time um, I struggled a little bit with the getting the blood to go up in the tube. And I think that they've changed that. I think it was just a drip, and that was, that made it way more easy. Way simpler because the straw, your little straw didn't work for me the first time

When I did it the first time I remember the tube was just very, very tiny, and it took a good couple of tries just to, you know, get all of the blood in there...I know the second time around that I did it, you were just able to kind of lift the cap, put it in there, and then close it and go. So that actually worked better the second time around 
Table 4 (continued)

\begin{tabular}{|c|c|c|}
\hline Stage of process & Theme & Illustrative quotations ${ }^{\mathrm{a}}$ \\
\hline & $\begin{array}{l}\text { Challenges with blood collection were associated with } \\
\text { unusual or incomplete test results }\end{array}$ & $\begin{array}{l}\text { The first time I remember [TelePrEP pharmacist] saying } \\
\text { my kidneys looked weird but it was just because I didn't } \\
\text { provide enough blood for it, it just wasn't working } \\
\text { I don't know why, I could never fill, whether I was dehy- } \\
\text { drated or, you know, the blood would not wick up into } \\
\text { that tube. I could never get enough blood into the blood } \\
\text { draw for them to get a decent sample }\end{array}$ \\
\hline \multirow[t]{2}{*}{ Return of kit } & Return shipping process was convenient & $\begin{array}{l}\text { The only thing that was a little bit hard to get around } \\
\text { was that because it had like bio-fluids in it, I couldn't } \\
\text { just give it back to our mailroom to send out. I had } \\
\text { to actually bring it into a, like a FedEx or UPS store. } \\
\text { Which still is more convenient because those are more } \\
\text { prominent than labs are } \\
\text { I have one right at work, and so I just took it the next } \\
\text { morning and dropped it in the box }\end{array}$ \\
\hline & Delays in kit return could affect test results & $\begin{array}{l}\text { I did the results at night, and by the time I got to [FedEx } \\
\text { location] it was closed. So I had to wait overnight to } \\
\text { ship it. But when that happened, I let [TelePrEP phar- } \\
\text { macist }] \text { know so she was aware of it, and aware of how } \\
\text { any results might be affected } \\
\text { The first one was, I had a, I did the home test kit and there } \\
\text { was some sort of mailing delay before it got to the lab } \\
\text { and my kidney function was elevated, I believe }\end{array}$ \\
\hline
\end{tabular}

Of twenty-one total in-depth interview participants, ten accepted the offer of a home kit

${ }^{a}$ Quotations have been lightly edited to remove word repetitions (e.g., stammering) and verbal hesitations (e.g., um), and to protect confidentiality

Interview data identified three main themes influencing client decision: self-efficacy, time/convenience, and privacy/ confidentiality. IDI participants reported they were likely to accept the offer of a kit if they had high self-efficacy to complete kit activities, viewed laboratory site visits as burdensome in terms of time, cost, and impact on schedule, and felt home kit use posed less risk to privacy and confidentiality. Clients often cited multiple factors and unique contextual considerations giving relative weight to those factors, rather than attributing their decision to one single factor. IDI participants reported several difficulties with blood collection - a process that was improved through client feedback and modified collection methods - but most kit users were able to have their prescriptions renewed based on specimens collected at home.

Access to required laboratory monitoring is an essential component of long-term PrEP adherence. Travel time, transportation access, stigma, and insurance status, among other factors, increase the testing burden on clients [7, 20]. Strategies addressing barriers to laboratory monitoring, such as home specimen self-collection, may increase PrEP uptake and adherence and decrease loss across the PrEP care cascade [10, 21-23], particularly in rural or other areas characterized as PrEP deserts. However, our findings demonstrate the use of home kits is not a one-size-fits-all solution for patients in PrEP care, and support previous research indicating some patients may in fact prefer laboratory site visits $[24,25]$.
We covered the cost of home kits and testing in this quality improvement initiative using internal Iowa TelePrEP funds. Scale up of home specimen collection in routine practice will require strategies to pay for kits and specimen processing, ideally with minimal out-of-pocket costs to clients. Healthcare payers may cover costs of home kits in some cases, and healthcare systems may be able to further cover costs of home kits through cost offsets related to reduced staff time required in specimen collection.

The ongoing COVID-19 pandemic has highlighted the increasing importance of telehealth and home specimen selfcollection methods in preventive healthcare delivery. Shifting PrEP visits and specimen collection to the home setting can address both public mandates and clients' personal preferences to socially-distance and minimize physical interactions with the healthcare system. Optimizing the home specimen self-collection process for telehealth delivery of PrEP care not only has relevance for the current pandemic, but will be important in post-pandemic times when clients accustomed to this option may prefer to continue using home kits rather than returning to in-person laboratory monitoring.

While specimen self-collection has been implemented in a number of clinical or conventional laboratory settings, research on home self-collection of specimens to date has largely focused on acceptability rather than feasibility or implementation in real-world settings [26]. One exception is Siegler et al.'s 
[6] initial feasibility pilot study of the home kits utilized in the Iowa TelePrEP initiative. As in Siegler et al.'s pilot, Iowa TelePrEP clients experienced issues with blood collection (e.g., inadequate specimens, test accuracy issues), albeit at higher rates. However, we believe this discrepancy is related to the specimen collection method of a capillary tube process versus the 'gravity' method used in Siegler et al.'s study, as previous studies have also supported successful home blood collection for HIV testing [27, 28]. It is also important to note most Iowa TelePrEP clients used a home kit only 1-3 times during the study period. It is likely that user proficiency in specimen collection and subsequent test completion rates would improve with repeated use. In the future, it will be important to continue to optimize usability of home specimen self-collection kits for PrEP monitoring, and to adapt home kits for additional populations of PrEP users such as cisgender women.

\section{Limitations}

Iowa TelePrEP offered clients the option of a home kit as a quality improvement initiative delivered during routine PrEP care processes in a low-population, predominantly rural state. As a result, our sample size was small, with limited demographic and geographic diversity, and there was no control group. Clients who chose to use home kits were selfselected. Furthermore, the interview sample was relatively small and homogenous; results may not be generalizable to broader populations in PrEP care.

\section{Conclusion}

Home specimen self-collection with central laboratory testing, when combined with innovative models of PrEP delivery such as telehealth, has the potential to reduce testing burden on clients and increase long-term persistence with PrEP. Telehealth programs should offer PrEP clients a choice of home kits or use of laboratory sites, as client preferences vary, and work with clients to ensure that processes for selfcollection of blood samples are simple and easy to follow, and that clients are supported in kit completion and return.

\section{Appendix}

\section{Appendix A}

See Fig. 2

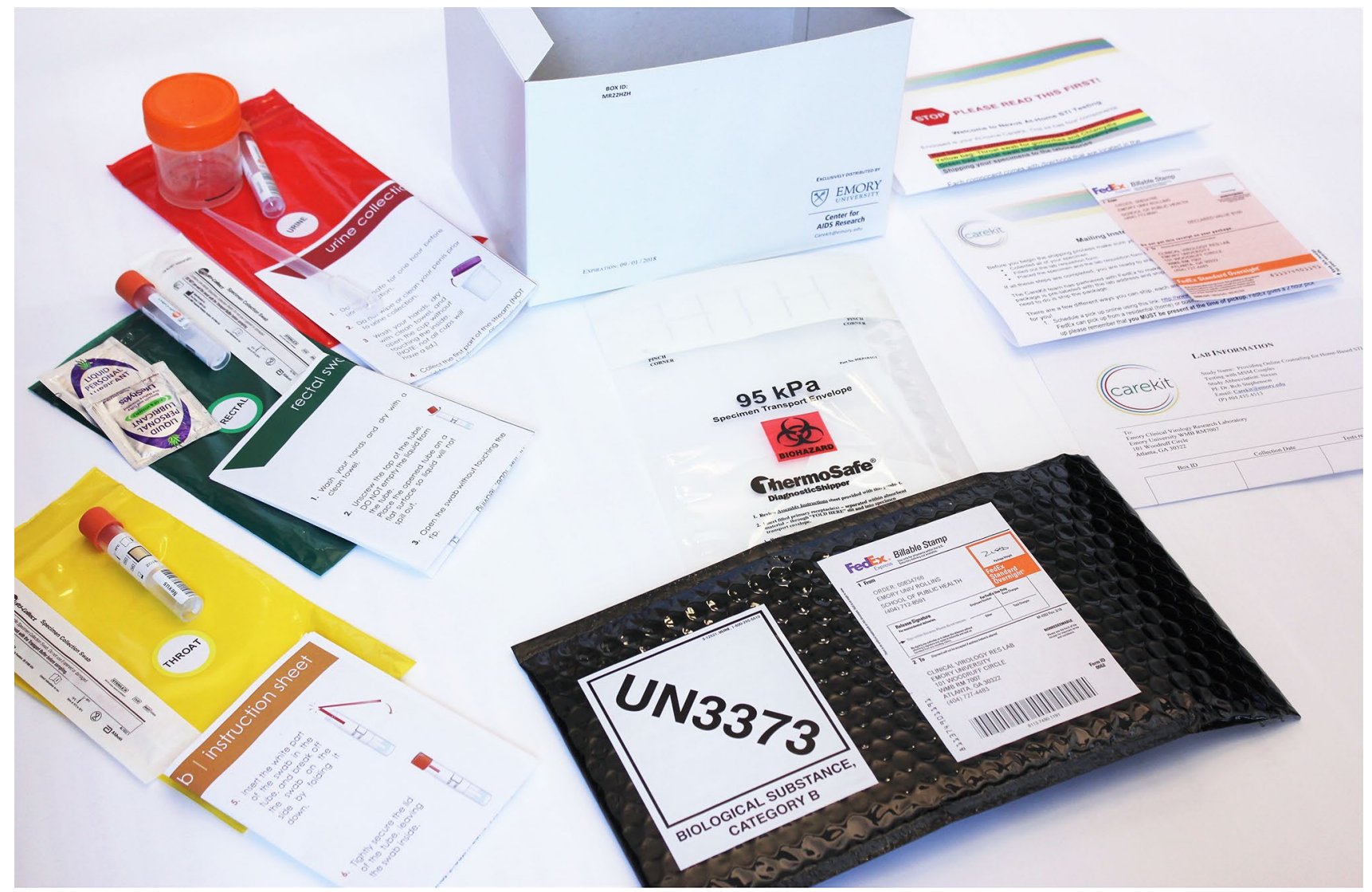

Fig. 2 Home kit contents 


\section{Appendix B}

See Fig. 3

\section{blood collection | instruction sheet (side 1)}

\section{START YOUR BLOOD COLLECTION HERE}

Visit https://vimeo.com/234695364/058428ef54 for video instructions

\section{A. SETTING UP}

1. Place the kit contents on their matching images in the "WORKING AREA" on this placemat.

2. Wash your hands with soap and warm water. To get blood flowing, leave your hands under warm water for 1 minute.

Dry with a clean towel.

3. Place the tube in the holder. Twist the cap off the tube and put the cap aside.

4. Select the finger to prick.

Choose the middle or ring finger from your non-dominant (weak) hand.

5. Choose a puncture site off center of your fingertip.

6. Twist cap off lancet and put aside. A small blade is inside the lancet and will be auto-activated at a later step.

7. Open alcohol wipe and gauze pad. Wipe selected fingertip with alcohol wipe.

8. Vigorously shake your hand below the waist for 15 seconds while standing to get blood flowing.
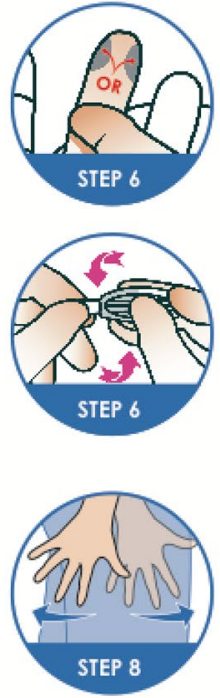

DESIGNED BY: LRDG - LRDESIGNGHOUPCON

\section{B. COLLECT BLOOD IN THE TUBE}

9. Press the lancet tip firmly against your finger until you hear a click.

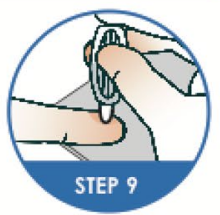

10. With a milking action, firmly squeeze your finger at the base and slide up until a large drop of blood forms.

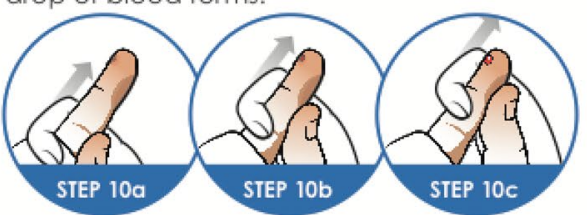

11. Turn your hand over and wipe the blood drop on the inside of the tube.

12. Usually the first few drops of blood will stick to the top and side of the tube. Keep going, and try getting bigger drops of blood. Your blood will eventually drop into the tube.
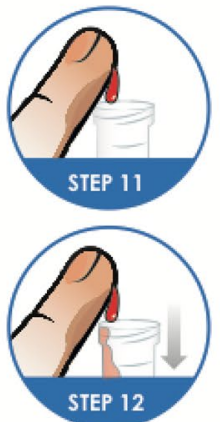

13. Fill tube to the black line. This may take 6-12 drops.

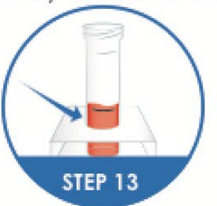

To increase blood flow:

a. Make a solid swipe with gauze pad to get blood flowing

b. Run your hand

under warm water.

c. If needed, use

second safely lancet.

If unable to fill the tube, call the Care Kit Support Center at (404) 435-4513.

14. Put filled tube aside.

TURN OVER AND CONTINUE TO STEP $15 \longrightarrow$

Fig. 3 Original home kit blood collection instructions 


\section{blood collection | instruction sheet (side 2)}

\section{FINISHING UP}

15. Use gauze pad to wipe remaining blood off your finger. Apply bandage.

16. Push the cap firmly onto the tube until it pops in place. The cap does not screw on.

17. Gently mix the blood in the tube, moving around so that the blood comes into contact with all sides of the tube.

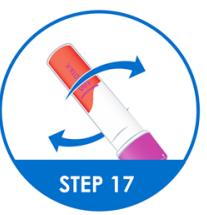

18. Place the tube in the plastic bag from other samples from previous steps, seal bag and place safely aside.

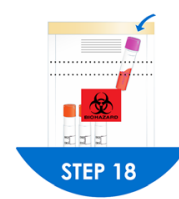

19. You can safely dispose of the lancets, alcohol wipe, and gauze pads in your home garbage.
If at any time you have questions, please call

the Care Kit Support Center at (404) 435-4513.

Your call is completely private and confidential.

Once you are done collecting all required specimen, place the biohazard bag and the completed lab requisition form in the bubble mailer and see the mailing instructions insert. If you have any questions, contact a CareKit team member at Carekit@emory.edu or 404.435.4513.

Fig. 3 (continued) 


\section{Appendix C}

See Fig. 4

\section{blood collection | instruction sheet (side 1)}

\section{START YOUR BLOOD COLLECTION HERE}

Visit https://vimeo.com/234695364/058428ef54 for video instructions

\section{A. SETTING UP}

1. Place the kit contents on their matching images in the "WORKING AREA" on this placemat.

2. Wash your hands with soap and warm water. To get blood flowing, leave your hands under warm water for 1 minute.

Dry with a clean towel.

3. Twist off top with thin capillary tube and throw away.

4. Select the finger to prick.

Choose the middle or ring finger from your non-dominant (weak) hand.

5. Choose a puncture site off center of your fingertip.

6. Twist cap off lancet and put aside. A small blade is inside the lancet and will be auto-activated at a later step.

7. Open alcohol wipe and gauze pad. Wipe selected fingertip with alcohol wipe.

8. Vigorously shake your hand below the waist for 15 seconds while standing to get blood flowing.
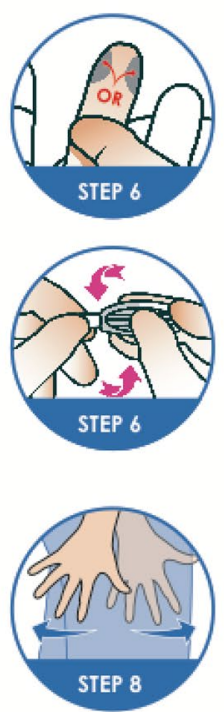

DESIGNED BY: LRDG LRDESIGNGROUP.COM

\section{B. COLLECT BLOOD IN THE TUBE}

9. Press the lancet tip firmly against your finger until you hear a click.

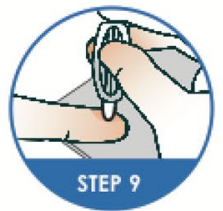

10. With a milking action, firmly squeeze your finger at the base and slide up until a large drop of blood forms.

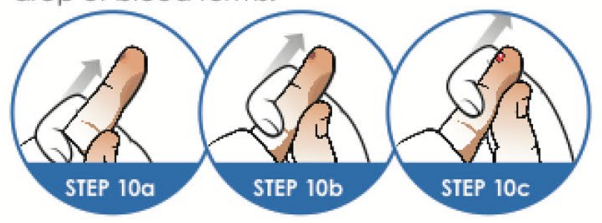

11. Turn your hand over and wipe the blood drop on the inside of the tube.

12. Usually the first few drops of blood will stick to the top and side of the tube. Keep going, and try getting bigger drops of blood. Your blood will eventually drop into the tube.
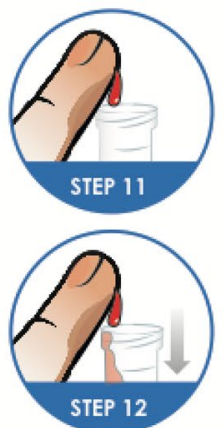

13. Fill tube to the black line. This may take 6-12 drops.

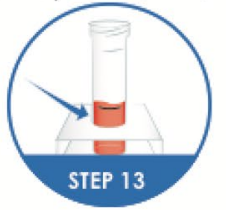

To increase blood flow:

a. Make a solid swipe

with gauze pad to

get blood flowing

b. Run your hand

under warm water.

c. If needed, use

second safety

lancet.

If unable to fill the fube, call the Care Kit Support Center at (404) 435-4513.

14. Put filled tube aside.

TURN OVER AND CONTINUE TO STEP $15 \longrightarrow$

Fig. 4 Revised home kit blood collection instructions 


\section{blood collection | instruction sheet (side 2)}

\section{FINISHING UP}

15. Use gauze pad to wipe remaining blood off your finger. Apply bandage.

16. Remove the purple cap from the base of the tube and seal the top. Push down until it clicks.

17. Gently mix the blood in the tube, moving around so that the blood comes into contact with all sides of the tube.

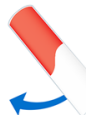

18. Place the tube in the plastic bag from other samples from previous steps, seal bag and place safely aside.

19. You can safely dispose of the lancets, alcoho wipe, and gauze pads in your home garbage.
If at any time you have questions, please call the Care Kit Support Center at (404) 435-4513.

Your call is completely private and confidential.

Once you are done collecting all required specimen, place the biohazard bag and the completed lab requisition form in the bubble mailer and see the mailing instructions insert. If you have any questions, contact a CareKit team member at Carekit@emory.edu or 404.435.4513.

Fig. 4 (continued) 
Acknowledgements The home kit initiative was supported by internal program funds at the University of Iowa, obtained through an award for innovation granted to Iowa TelePrEP by the American Academy of HIV Medicine. The initiative was also supported in part by the National Institute of Mental Health (RO1MH114692) and by the Emory Center for AIDS Research (P30AI050409). The content is solely the responsibility of the authors and does not necessarily represent the official views of the National Institutes of Health. The authors are grateful to all our participants for their involvement in the home kit initiative and evaluation.

\section{Declarations}

Conflict of interest Dr. Ohl has received research support from and performed consulting for Gilead Sciences, Inc., unrelated to the work presented here. Dr. Hoth has performed consulting for Gilead Sciences, Inc., unrelated to the work presented here. Dr. Siegler is an investigator on a grant from Gilead Foundation, paid to his institution.

Ethical approval Approval was obtained from the Institutional Review Board of the University of Iowa. The procedures used in this study adhere to the tenets of the Declaration of Helsinki.

Informed consent Informed consent was obtained prior to interviews.

\section{References}

1. Baeten JM, Donnell D, Ndase P, Mugo NR, Campbell JD, Wangisi $\mathrm{J}$, et al. Antiretroviral prophylaxis for HIV prevention in heterosexual men and women. N Engl J Med. 2012;367(5):399-410.

2. Choopanya K, Martin M, Suntharasamai P, Sangkum U, Mock PA, Leethochawalit M, et al. Antiretroviral prophylaxis for HIV infection in injecting drug users in Bangkok, Thailand (the Bangkok Tenofovir Study): a randomised, double-blind, placebo-controlled phase 3 trial. Lancet. 2013;381(9883):2083-90.

3. Grant RM, Lama JR, Anderson PL, McMahan V, Liu AY, Vargas $\mathrm{L}$, et al. Preexposure chemoprophylaxis for HIV prevention in men who have sex with men. N Engl J Med. 2010;363(27):2587-99.

4. Centers for Disease Control and Prevention: US Public Health Service: Preexposure prophylaxis for the prevention of HIV infection in the United States-2017 Update: a clinical practice guideline. 2018. https://www.cdc.gov/hiv/pdf/risk/prep/cdc-hivprep-guidelines-2017.pdf.

5. Calabrese SK. Interpreting gaps along the preexposure prophylaxis cascade and addressing vulnerabilities to stigma. Am J Public Health. 2018;108(10):1284-6.

6. Siegler AJ, Mayer KH, Liu AY, Patel RR, Ahlschlager LM, Kraft $\mathrm{CS}$, et al. Developing and assessing the feasibility of a homebased preexposure prophylaxis monitoring and support program. Clin Infect Dis. 2019;68(3):501-4.

7. Sullivan PS, Mena L, Elopre L, Siegler AJ. Implementation strategies to increase PrEP uptake in the South. Curr HIV/AIDS Rep. 2019;16(4):259-69.

8. Hubach RD, Currin JM, Sanders CA, Durham AR, Kavanaugh $\mathrm{KE}$, Wheeler DL, et al. Barriers to access and adoption of preexposure prophylaxis for the prevention of HIV among men who have sex with men (MSM) in a relatively rural state. AIDS Educ Prev. 2017;29(4):315-29.

9. Calabrese SK, Underhill K. How stigma surrounding the use of HIV preexposure prophylaxis undermines prevention and pleasure: a call to destigmatize "Truvada Whores." Am J Public Health. 2015;105(10):1960-4.

10. Whitfield THF, John SA, Rendina HJ, Grov C, Parsons JT. Why I quit pre-exposure prophylaxis (PrEP)? A mixed-method study exploring reasons for PrEP discontinuation and potential re-initiation among gay and bisexual men. AIDS Behav. 2018;22(11):3566-75.

11. Refugio ON, Kimble MM, Silva CL, Lykens JE, Bannister C, Klausner JD. PrEPTECH: a telehealth-based initiation program for human immunodeficiency virus pre-exposure prophylaxis in young men of color who have sex with men A pilot study of feasibility. J Acquir Immune Defic Syndr. 2019;80(1):40-5.

12. Hoth AB, Shafer C, Dillon DB, Mayer R, Walton G, Ohl ME. Iowa TelePrEP: a public-health-partnered telehealth model for human immunodeficiency virus preexposure prophylaxis delivery in a rural state. Sex Transm Dis. 2019;46(8):507-12.

13. Stekler JD, McMahan V, Ballinger L, Viquez L, Swanson F, Stockton J, et al. HIV pre-exposure prophylaxis prescribing through telehealth. J Acquir Immune Defic Syndr. 2018;77(5):e40-2.

14. Siegler AJ, Brock JB, Hurt CB, Ahlschlager L, Dominguez K, Kelley CF, et al. An electronic pre-exposure prophylaxis initiation and maintenance home care system for nonurban young men who have sex with men: protocol for a randomized controlled trial. JMIR Res Protoc. 2019;8(6):e13982.

15. SAS Institute, Inc. SAS software. 9.4 ed. Cary, NC: SAS Institute, Inc.; 2013.

16. R Core Team. R: A Language and Environment for Statistical Computing. Vienna, Austria: R Foundation for Statistical Computing; 2019.

17. VERBI Software. MAXQDA 2020. 20.0.06 ed. Berlin, Germany: VERBI Software; 1995-2020.

18. Brooks J, King, N. Doing template analysis: evaluating an endof-life care service. In: SAGE Research Methods Cases. London: SAGE Publications; 2014.

19. King N. Using templates in the thematic analysis of text. In: Catherine C, Gillian S, editors. Essentials Guide to Qualitative Methods in Organizational Research. London: SAGE Publications Ltd; 2004. p. 256-70.

20. Siegler AJ. Needed: a life course perspective on maintaining PrEP use. Clin Infect Dis. 2020;72(3):386-7.

21. Kelley CF, Kahle E, Siegler A, Sanchez T, Del Rio C, Sullivan PS, et al. Applying a PrEP continuum of care for men who have sex with men in Atlanta. Georgia Clin Infect Dis. 2015;61(10):1590-7.

22. Nunn AS, Brinkley-Rubinstein L, Oldenburg CE, Mayer KH, Mimiaga M, Patel R, et al. Defining the HIV pre-exposure prophylaxis care continuum. AIDS. 2017;31(5):731-4.

23. Parsons JT, Rendina HJ, Lassiter JM, Whitfield TH, Starks TJ, Grov C. Uptake of HIV pre-exposure prophylaxis (PrEP) in a national cohort of gay and bisexual men in the United States. J Acquir Immune Defic Syndr. 2017;74(3):285-92.

24. Koester KA, Hughes SD, Grant RM. "A good habit": telehealth PrEP users find benefit in quarterly monitoring requirements. J Int Assoc Provid AIDS Care. 2020;19:232595822091926.

25. John SA, Rendina HJ, Grov C, Parsons JT. Home-based pre-exposure prophylaxis (PrEP) services for gay and bisexual men: An opportunity to address barriers to PrEP uptake and persistence. PLoS ONE. 2017;12(12):0189794.

26. Sharma A, Stephenson R, Sallabank G, Merrill L, Sullivan S, Gandhi M. Acceptability and feasibility of self-collecting biological specimens for HIV, sexually transmitted infection, and adherence testing among high-risk populations (Project Caboodle!): 
protocol for an exploratory mixed-methods study. JMIR Res Protoc. 2019;8(5):e13647.

27. Ricca AV, Hall EW, Khosropour CM, Sullivan PS. Factors associated with returning at-home specimen collection kits for HIV testing among internet-using men who have sex with men. J Int Assoc Provid AIDS Care. 2016;15(6):463-9.

28. MacGowan RJ, Chavez PR, Borkowf CB, Owen SM, Purcell DW, Mermin JH, et al. Effect of internet-distributed HIV self-tests on HIV diagnosis and behavioral outcomes in men who have sex with men: a randomized clinical trial. JAMA Intern Med. 2020;180(1):117-25.

Publisher's Note Springer Nature remains neutral with regard to jurisdictional claims in published maps and institutional affiliations. 